\title{
Protective Effect of Chinonin in MPTP-Induced C57BL/6 Mouse Model of Parkinson's Disease
}

\author{
Guoshuai Feng, ${ }^{a, \#}$ Zhijian Zhang, ${ }^{a, \#}$ Qingqing Bao, ${ }^{b}$ Zaijun Zhang, ${ }^{a}$ Libing Zhou, ${ }^{c}$ \\ Jie Jiang, ${ }^{*, a, e}$ and Sha $\mathrm{Li}^{*}, d$ \\ ${ }^{a}$ College of Pharmacy, Jinan University; ${ }^{d}$ Department of Pharmaceutics, College of Pharmacy, Jinan University; \\ Guangzhou 510632, China: ${ }^{b}$ The Children's Hospital Zhejiang University School of Medicine; Hangzhou 310006, \\ China: ${ }^{c}$ The Joint Laboratory for Brain Function and Health (BFAH), Jinan University and The University of Hong \\ Kong, Medical School of Jinan University; Guangzhou 510632 China: and ${ }^{e}$ DongGuan Institute of Jinan University; \\ DongGuan 523808, China. \\ Received February 7, 2014; accepted March 31, 2014; advance publication released online May 28, 2014
}

The aims of this study were to investigate the effect of chinonin in preventing 1-methyl-4-phenyl-1,2,3,6tetrahydropyridine (MPTP)-induced neurodegeneration in C57BL/6 mice and to examine the possible mechanisms. The neurotoxin MPTP was employed to create a subacute Parkinson's disease (PD)-like model in C57BL/6 mice. Chinonin $(10,20,40 \mathrm{mg} / \mathrm{kg}$ body weight) was intraperitoneally administered $0.5 \mathrm{~h}$ after MPTP $(30 \mathrm{mg} / \mathrm{kg})$ injection for $7 \mathrm{~d}$ consecutively. Chinonin showed neuroprotective effects in the MPTP-treated mice PD model by ameliorating motor impairment in the catwalk and open-field tests. Consistently, chinonin reduced loss of dopaminergic neurons in the substantia nigra and prevented depletion of dopamine and its metabolites 3-methoxy-4-hydroxy-phenylacetic acid and homovanillic acid in the striatum of mice. Compared with the MPTP group, in the chinonin plus MPTP groups significant increases of superoxide dismutase activity and glutathione levels were observed as well as a distinct reduction of lipid peroxidation product malondialdehyde in the striatum. Taken together, we propose that chinonin exerts neuroprotective effects in C57BL/6 mouse model of PD and these effects may be due to chinonin's antioxidative property.

Key words chinonin; Parkinson's disease (PD); neuroprotection; behavior; oxidative stress

Parkinson's disease (PD) is a major neurodegenerative disorder characterized pathologically by a progressive loss of dopaminergic neurons and clinically by resting tremors, rigidity, slowness of movement and postural instability. ${ }^{1)}$ Systemic administration of 1-methyl-4-phenyl-1,2,3,6-tetrahydropyridine (MPTP), produces neuropathological and clinical hallmarks in humans, monkeys and mice that closely resemble idiopathic parkinsonism. ${ }^{2,3)}$ MPTP is transformed to the 1-methyl4-phenylpyridinium ion $\left(\mathrm{MPP}^{+}\right)$by monoamine oxidase type $\mathrm{B}$ after administration. ${ }^{4}$ ) Then $\mathrm{MPP}^{+}$is selectively absorbed by the dopaminergic neurons in the substantial nigra via the dopamine transporter and impairs mitochondrial respiration by inhibiting complex I, thereby blocking the production of ATP and lead to the production of reactive oxygen species (ROS). ${ }^{5)}$ Therefore, MPTP acted as a pro-oxidant in the progress of PD in MPTP-induced mouse.

Although the etiologic mechanisms of PD are still obscure, oxidative injury is considered as a pivotal role in disease pathogenesis. $^{6,7)}$ Chinonin (Fig. 1), a natural multi-phenols compound also named Mangifera indica, has been reported to possess anti-oxidative, anti-inflammatory, anti-diabetic, anti-platelet aggregator, antiviral, and anti-depressant properties. ${ }^{8)}$ Given its anti-oxidative property, chinonin have been extensively used in the Indian sub-continent as food additives and in cosmetics and medicines. ${ }^{9}$ Besides, chinonin is able to cross the blood brain barrier and has the real potential to ameliorate the oxidative stress observed in neurodegenerative disorders. ${ }^{10,11)}$ Its antioxidant properties have been extensively evaluated in various cell lines, including neurons. ${ }^{12)}$ For example, Amazzal found that chinonin can protects against $\mathrm{MPP}^{+}$

The authors declare no conflict of interest.

\# These authors contributed equally to this work. toxicity mediated by oxidative stress in N2A cells. ${ }^{13)}$

In the present study, we investigated the effects of chinonin on MPTP-induced dopaminergic neuron damage and behavioral disorder in C57BL/6 mice. We found chinonin significantly attenuated the serious behavioral disorder, reduced the death of substantia nigra neurons and reversed the levels of dopamine (DA) and its metabolites 3-methoxy-4-hydroxyphenylacetic acid (DOPAC) and homovanillic acid (HVA) in the striatum of MPTP-induced mice. As an inhibitor of monoamine oxidase B, anti-parkinsonism drug selegiline is also an antioxidant, so we use selegiline as positive control here.

\section{MATERIALS AND METHODS}

Reagents MPTP and selegiline (deprenyl, sele) were obtained from Sigma-Aldrich (St. Louis, MO, U.S.A.). Chinonin (mangiferin, $>99 \%$ ) was extracted, isolated and purified from plant X. in China Resources by Sanjiu Medical \& Pharmaceutical Co., Ltd. The antibody against tyrosine hydroxylase was supplied by Millipore (Billerica, MA, U.S.A.) and anti-rabbit secondary antibody was supplied by Cell Signaling Technology (Beverly, MA, U.S.A.). Diaminobenzidin (DAB) staining solution was from Gene Tech Company, Limited (Shanghai,<smiles>O=C1c2cc(O)c(O)cc2OC2C=C(O)C(C3O[C@H](CO)[C@@H](O)[C@H](O)[C@H]3O)=C(O)C12</smiles>

Fig. 1. Structure of Chinonin 
China). Pierce bicinchoninic acid (BCA) protein assay kits was purchased from Thermo Scientific (Rockford, IL, U.S.A.). Glutathione (GSH), malondialdehyde (MDA) and superoxide dismutase (SOD) assay kits were purchased from Nanjing Jiancheng Bioengineering Institute (Nanjing, China). All other reagents were purchased from Sigma-Aldrich unless stated otherwise.

Animals and Treatment Male C57BL/6 mice, 8-10 weeks of age and weighing 20-22g, were obtained from Medical Experimental Animal Center of Guangdong province. All animals were housed at a constant temperature (24士 $\left.1{ }^{\circ} \mathrm{C}\right)$ and humidity-controlled $(60 \pm 10 \%)$ environment under a $12 \mathrm{~h}$ light/dark cycle and given food and water ad libitum. All the animal studies described in this paper were approved and conducted in accordance with the Guidelines of Laboratory Animal Ethics Committee of Jinan University (Approval ID: 20121116002).

Total 108 animals were divided into six groups containing 18 animals each. The groups included vehicle, MPTP $(30 \mathrm{mg} /$ $\mathrm{kg}$, MPTP + positive drug $(10 \mathrm{mg} / \mathrm{kg}$ selegiline), MPTP + chinonin $(10 \mathrm{mg} / \mathrm{kg}), \mathrm{MPTP}+$ chinonin $(20 \mathrm{mg} / \mathrm{kg})$, and MPTP+ chinonin $(40 \mathrm{mg} / \mathrm{kg})$. The mice of all groups except vehicle group were administered with MPTP- $\mathrm{HCl}$ before treated with different dose of chinonin or selegiline half an hour later, once a day. Mice of vehicle group were administered equal volume of solvent. For all the groups, compounds including MPTP$\mathrm{HCl}$, chinonin, selegiline were dissolved in same solvent (normal saline containing 1\% dimethyl sulfoxide (DMSO)) and administered intraperitoneally. The injection lasted for 7 consecutive days and behavior tests were carried out after the last injection. Then the striatum of mice were collected.

Catwalk The catwalk apparatus comprises a long glass plate with a fluorescent light beamed into the glass walkway floor from one side. In a dim environment, the light is reflected downward and the footprints of the animal as it walks along the walkway are recorded by a camera mounted under the glass. ${ }^{14)}$ Mice were trained to cross the walkway daily one week prior to the test. After last administarion, the mice performed uninterrupted runs for at least three times and the data were analyzed by using the automated gait analysis system Cat Walk (Noldus Information Technology, Wageningen, the Netherlands).

Open Field Test Spontaneous locomotor activity was evaluated by testing mice using an Open Field system $(50 \mathrm{~cm}$ in diameter, $40 \mathrm{~cm}$ in height) which was made of plywood with a frontal glass wall. The experiments were performed in a sound-attenuated room under low-intensity light. Animals were placed individually in the same corner, allowing the mice accustomed to the new environment for a few minutes, and their behavior was recorded on videotape for $10 \mathrm{~min}$. The open field was washed with ethanol solution $(10 \%)$ and dried between each test to remove odor trails. ${ }^{15)}$ The recorded behavior was analyzed by EthoVision Version 2.3 (Noldus Information Technology, Wageningen, the Netherlands).

Immunohistochemistry Examination Under anesthesia with $10 \%$ chloral hydrate, 6 mice of each group were first perfused with heparinized saline flush, followed by $4 \%$ paraformaldehyde in phosphate buffered saline (PBS). Then the brains were dehydrated and embedded in paraffin. Next, fixed brains were cut into $5 \mu \mathrm{m}$ using a microtome and the paraffin sections of substantia nigra pars compacta (SNpc) were used for immunohistochemistry examination. The sections were boiled in sodium citrate-hydrochloric acid buffer solution for antigen repairing before blocking for $1 \mathrm{~h}$ in PBS containing $10 \%$ fetal bovine serum (FBS). Then the sections were incubated in a solution of buffer $(0.3 \%$ bovine serum albumin (BSA), 1\% goat serum, 0.3\% PBST) containing hydroxylase primary antibody $(1: 500)$ overnight at $4^{\circ} \mathrm{C}$. After rinsing with PBS, the sections were incubated in a biotinylated secondary antibody $(1: 200)$ with horseradish peroxidase (HRP) for $2 \mathrm{~h}$ and then visualized with diaminobenzidin (DAB). At last, the sections were dehydrated through an alcohol series, cleared in xylene and coverslipped. The sections from the same position of brain were photographed $\times 20$ magnifications on a stereomicroscope (BX51, Olympus Corp., Japan). Dopaminergic neurons were determined manually by counting the $\mathrm{TH}$ positive cells in SNpc of the same side. TH-positive cells were counted manually by operator who was blinded to the experiment and a total of 10 sections of each animal were analyzed.

Measurement of Striatal DA, DOPAC and HVA Levels Six mice of each group were sacrificed and striatum were collected to detect the level of DA and its metabolites DOPAC and HVA. On the day of analysis, the weighed samples were thawed to room temperature and homogenized in perchloric acid $(0.3 \mathrm{~mol} / \mathrm{L})$ containing $0.01 \%$ ethylenediaminetetraacetic acid (EDTA). The homogenate was centrifuged at $12000 \mathrm{rpm}$ for $10 \mathrm{~min}$ at $4^{\circ} \mathrm{C}$. The supernate was filtered in $0.22-\mu \mathrm{m}$ filter membrane, and $20 \mu \mathrm{L}$ of the filtrate was injected for analysis. The level of DA, DOPAC and HVA in striatum was measured by HPLC coupled to a 2465 electrochemical detector (Waters, U.S.A.) with a potential set at $+0.45 \mathrm{~V}$.

Antioxidative Activity Evaluation by Biochemical Assay To assess the changes of oxidative injury indexes, 6 mice of each group were euthanized and their substantial nigra were dissected out and homogenized in PBS buffer. The samples were then centrifuged at $10000 \times \boldsymbol{g}$ for $15 \mathrm{~min}$ at $4^{\circ} \mathrm{C}$. The protein in the supernatant was quantified by BCA protein assay kit. GSH, MDA and SOD assay kits were used for measurement according to the manufacturer instructions respectively.

Statistical Analyses The results were expressed as mean \pm S.E.M. One way ANOVA was used, followed by Tukey's multiple comparison test. All statistical analyses were conducted using GraphPad Prism5 (GraphPad Software Inc., San Diego, CA, U.S.A.). The $p$ values $<0.05$ were considered statistically significant.

\section{RESULTS}

Effects of Chinonin on Gait Parameters in MPTP-Treated Mice Gait disturbances are one of the most common movement disorders in PD. The catwalk method is an automated and computerized gait-analysis technique that allows objective quantification of multiple gait parameters. ${ }^{16)}$ Nowadays, this method is acceptable for the behavioral evaluation in animal models of central nervous system based movement disorders. The gait parameters were described as follows. A: Stand (s) is the duration in seconds of contact of a paw with the glass plate. B: Swing (s) is the duration in seconds of no contact of a paw with the glass plate. C: Swing Speed $(\mathrm{cm} / \mathrm{s})$ is the speed of the paw during swing. D: Run Duration (s): the duration for animals through the walkway floor once. E: The Regularity Index (\%): the number of normal step sequence 


\section{Catwalk}
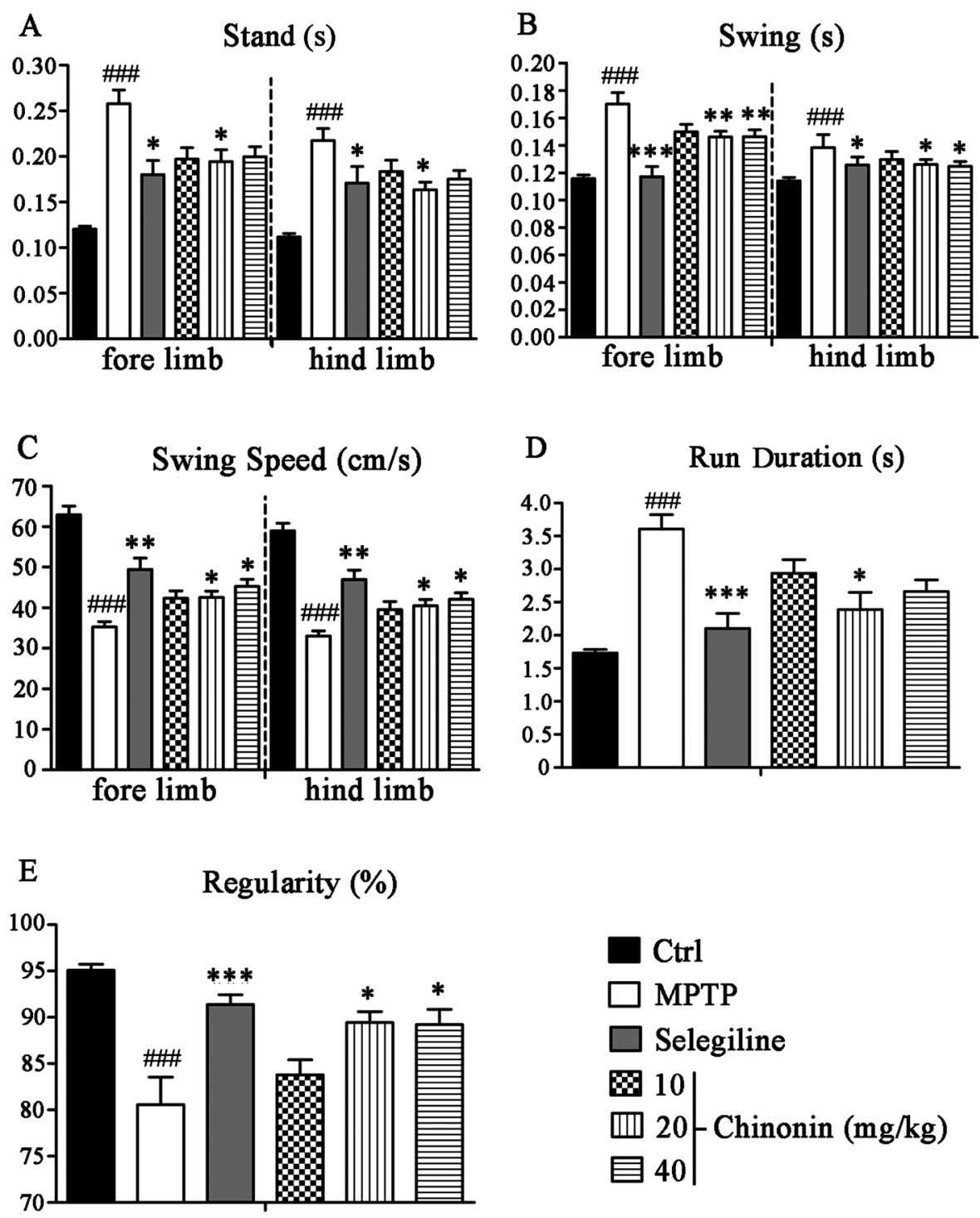

Fig. 2. Effect of Chinonin on Gait Deficits of MPTP-Treated Mice

Data were expressed as mean \pm S.D. $(n=18) .{ }^{\# \#} p<0.001$ compared to control group; $* p<0.05, * * p<0.01$ and $* * * p<0.001$ compared to MPTP-treated group.

patterns relative to the total number of paw placements.

As shown in Fig. 2, MPTP decreased the regularity index, swing speed and prolonged run-duration, stand and swing in model mice. The impairment of gait behaviors was ameliorated by chinonin treatment at $20 \mathrm{mg} / \mathrm{kg}$, but not at $10 \mathrm{mg} /$ $\mathrm{kg}$. Chinonin at the dose $40 \mathrm{mg} / \mathrm{kg}$ showed protective effect in swing, swing speed and regularity but not in stand and run duration.

MPTP-Induced Locomotor Dysfunction Was Improved in Chinonin Treated Mice To further assess the efficacy of chinonin against motor deficits induced by MPTP, more motor performance parameters were measured by open-field test. Representative motor activity maps of mice (Fig. 3A) indicated that chinonin helped MPTP-treated mice recover their mobility. The statistical results (Fig. 3B-D) showed MPTP lead to decrease of moved distance, ambulation time as well as the frequency in center, and chinonin at different dose im- proved these locomotor dysfunctions.

The results of catwalk and open field indicated chinonin could restored behavioral impairments caused by MPTP intoxication and normalize the abnormal behavior of MPTPtreated mice.

MPTP-Induced Neurotoxicity of SNpc Dopaminergic Neuron is Blocked by Chinonin Treatment with the neurotoxin MPTP resulted in a loss of TH-positive neurons in SNpc, which parallels the DA neuron loss seen in PD. ${ }^{17)}$ Representative microphotographs of $\mathrm{TH}$ immunostaining in the SNpc proved chinonin's neuroprotective effect (Fig. 4A). Statistical results demonstrated MPTP produced approximately $60 \%$ of neuron loss in SNpc. In contrast, chinonin at 10, 20 and $40 \mathrm{mg} / \mathrm{kg}$ significantly rescued the MPTP-induced loss of TH-positive neurons (Fig. 4B). The results suggested chinonin blocked MPTP-induced neurotoxicity of SNpc dopaminergic neuron in mice. 


\section{Open Field}

A
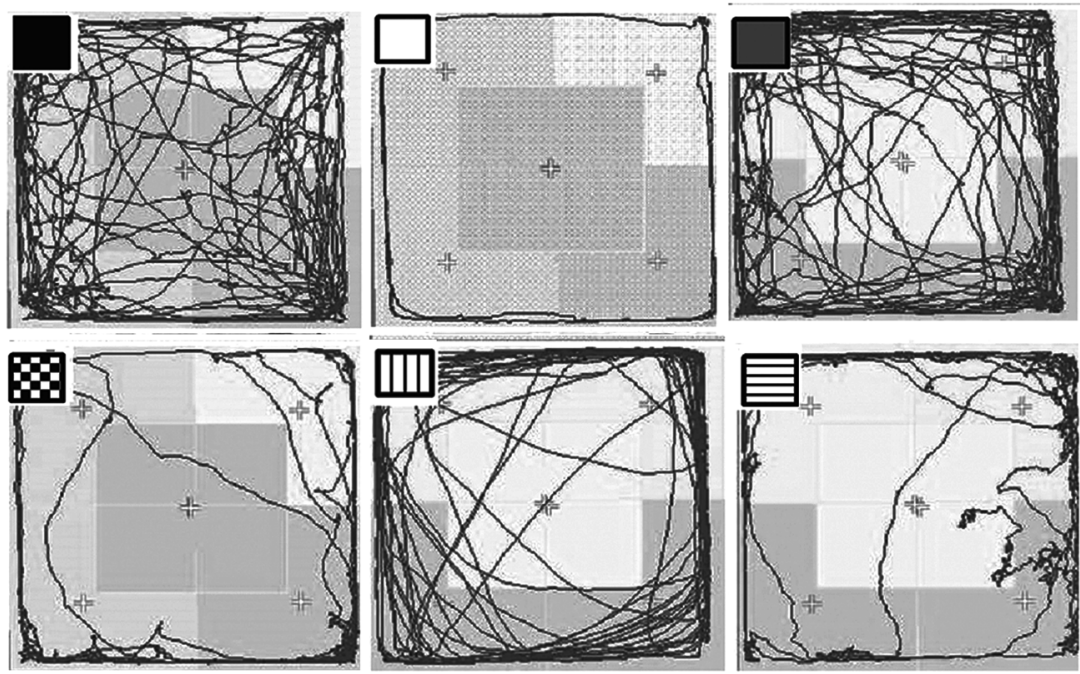

B

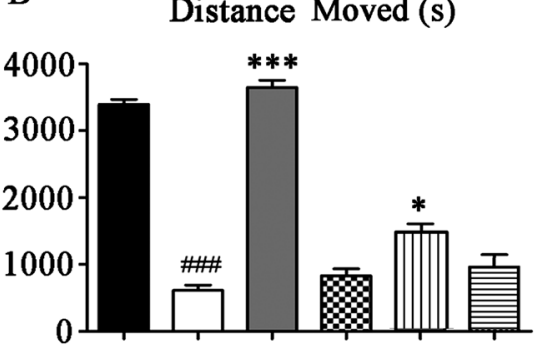

D

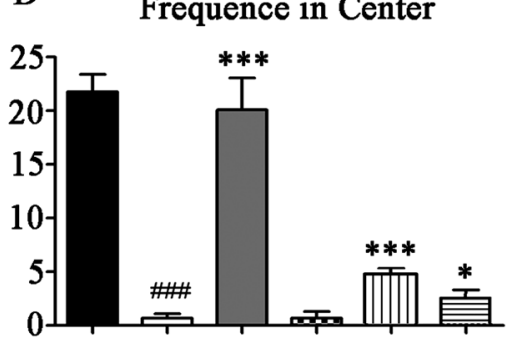

C Velocity $(\mathrm{cm} / \mathrm{s})$
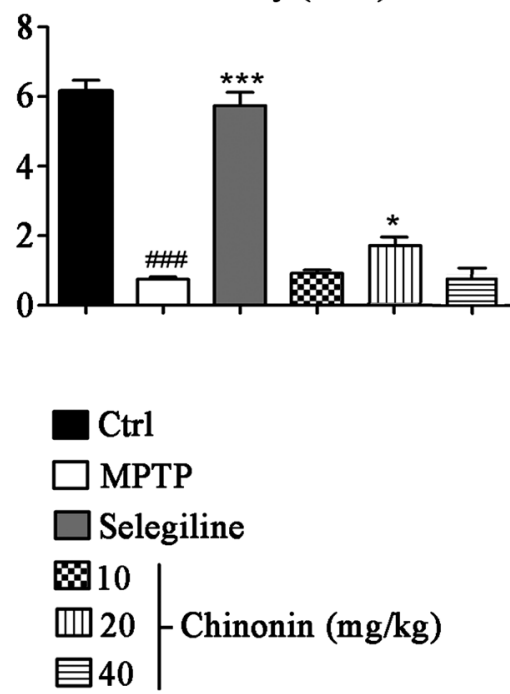

Fig. 3. Effect of Chinonin on Total Distance, Velocity and Frequence in Center Zone in the Open Field Test and Representative Maps of Mice Activities

Data were expressed as mean \pm S.D. $(n=18) .{ }^{\# \#} p<0.001$ compared to control group; $* p<0.05$ and $* * * p<0.001$ compared to MPTP-treated group.

Chinonin Attenuated Depletion of DA and Its Metabolites in the Striatum Loss of dopaminergic neurons lead to the depletion of DA and its metabolites. MPTP treatment significantly reduced the levels of striatal DA, DOPAC, and HVA. Chinonin at different dosages rescued the MPTP-induced loss of DA, DOPAC and HVA to different extend. As shown in Fig. 5, chinonin attenuated the MPTP-induced decrease of DA and its metabolites DOPAC and HVA in striatum which was consistent with the results of immunohistochemistry.

Chinonin Reversed the Overproduction of MDA and Increased the Level of GSH and SOD MPTP destroys the defense of anti-oxidant enzymes and elevates lipid peroxidation level in mice brain. ${ }^{18)}$ MDA was the stable metabolite of lipid peroxidation products. GSH was an important antioxidant and SOD was a pivotal anti-oxidant enzyme in the body. Our re- sults suggested that MPTP significantly led to the overproduction of MDA, the reduction of GSH and the decrease of SOD activity. Compared with MPTP group, chinonin at $20 \mathrm{mg} / \mathrm{kg}$ decreased production of MDA by $24.1 \%$ and increased the production of GSH by $126.8 \%$; and increased the activity of SOD by $14.8 \%$ and $33.4 \%$ at the dose of $10 \mathrm{mg} / \mathrm{kg}$ and $20 \mathrm{mg} /$ $\mathrm{kg}$, respectively (Fig. 6). The results indicated a powerful antioxidative capacity of chinonin in MPTP treated mice.

\section{DISCUSSION}

In our present work, we assessed chinonin's neuroprotective effect in animal PD models. We demonstrated that chinonin's neuroprotection against MPTP-induced mice's motor deficits, nigrostriatal degeneration and oxidative stress. Mice 
A
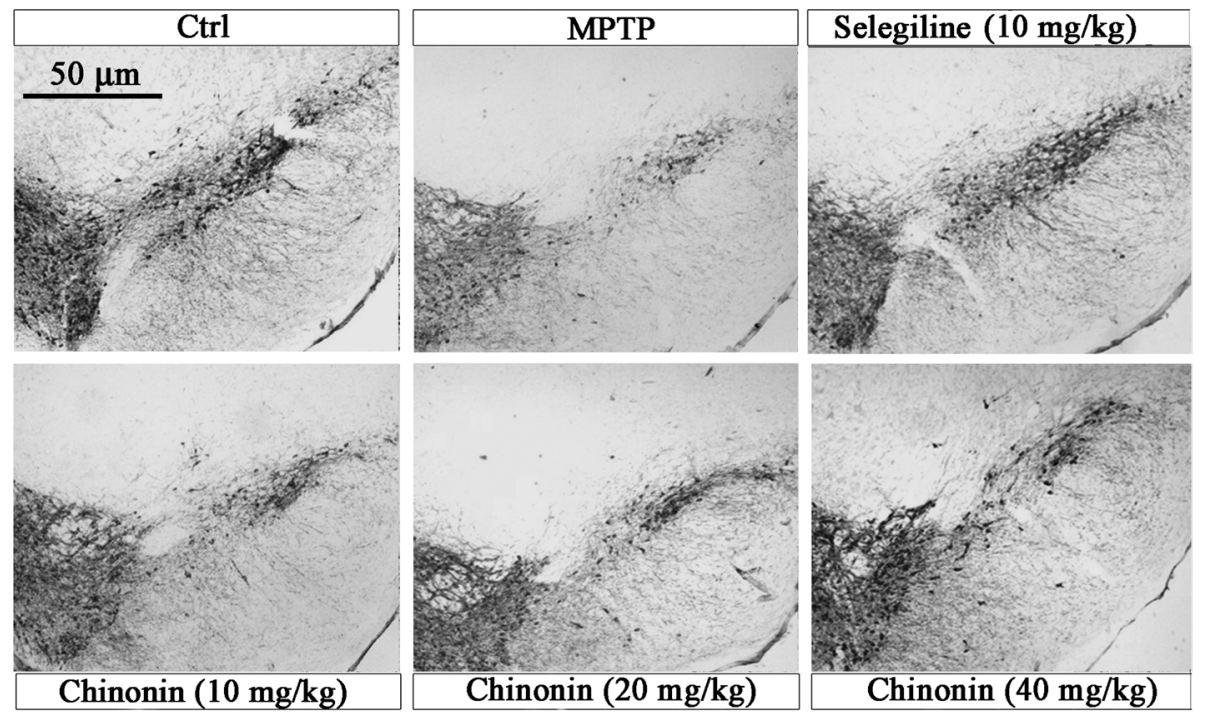

B

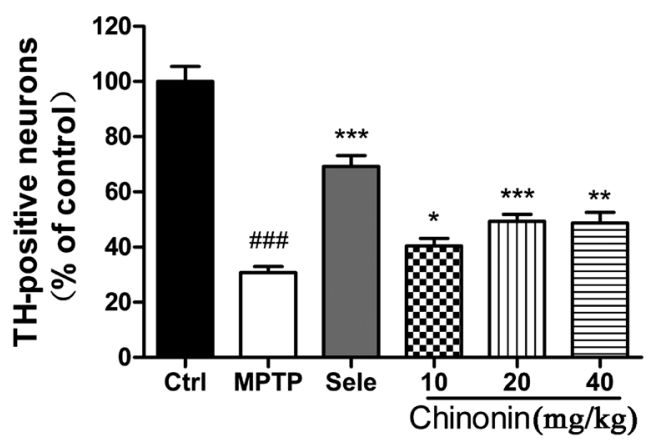

Fig. 4. Effect of Chinonin on Damage of TH-Positive Cells in the Substantial Nigra of MPTP-Treated Mice

(A) Representative microphotographs of TH immunostaining mouse brain sections $(20 \times)$. (B) Quantification of TH-positive neurons. Data were expressed as mean \pm S.D. $(n=6) .{ }^{\# \#} p<0.001$ compared with the control group; $* p<0.05, * * p<0.01$ and $* * * p<0.001$ compared with the MPTP-treated group.

performed a series of PD behaviour after injection of MPTP, such as incoordination, tremble, stiffness and bradykinesia. Gait change is considered as a useful indicator of severity in animal models of PD. So as a systematic and extensive gait analysis, catwalk was applied in our experiment. Open field was also used to evaluate neurological deficits of mice. Chinonin significantly helped MPTP-induced mice recover their mobility, including range of activity, movement speed, and limb strength. The decrease of TH-positive neurons reflects the severity of PD disease and the immunohistochemistry results were generally in accordance with behaviors. HPLC results also indicated chinonin blocked MPTP-induced loss of striatal DA, DOPAC and HVA.

Chinonin might have a little action in increasing DA secretion or preventing DA degradation, thus its action in enhancing DA level was lower than selegiline which is a selective monoamine oxidase inhibitor, while the metabolites level of DA in mice treated with chinonin/MPTP was comparable to or somewhat higher than those treated with selegiline/MPTP. The TH-positive neuron protection might account for the higher level of DA and its metabolites in chinonin/MPTP treated mice when compared with mice in MPTP treated model control group. On the other hand, the metabolic process is generally complicated, the DA levels may not strictly consistent with the level of its metabolites, Guo et al. found similar phenomenon in their study of tetramethylpyrazine nitrone. ${ }^{19}$ Meanwhile, the results of behavior test should be, but not always consistent with the results of immunohistochemistry, as shown in the results of Figs. 2-4.

Oxidative injury plays an important role in the pathogenesis of PD. Recent studies found cell injury during progress of PD are associated with overproduction of ROS. ${ }^{20)}$ Numerous studies on postmortem brain tissues of PD patients have suggested that ROS are involved in the degeneration of dopaminergic neurons. ${ }^{21)}$ This is primarily due to high levels of lipid peroxidation, depletion of GSH and increased protein oxidation in brain tissues of PD patients. ${ }^{22)}$ In our study, chinonin showed high clearance on lipid peroxidation product MDA and reversed the decrease of the anti-oxidant GSH in MPTP treated mice. These results were in agreement with previous reports suggesting the role of oxidative stress in neuronal cell death associated with $\mathrm{PD}^{23)}$

Most of our results in locomotor dysfunction improvement, protection of TH-positive neuron, DA level enhancement and anti-oxidative activity showed a similar dose-effect relationship, with the moderate dose $20 \mathrm{mg} / \mathrm{kg}$ demonstrating the 
A

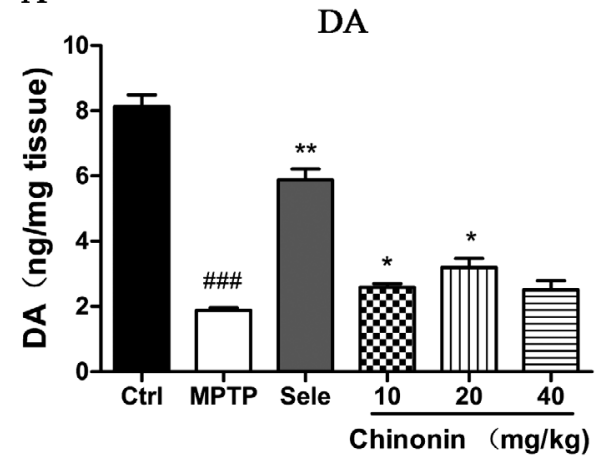

c

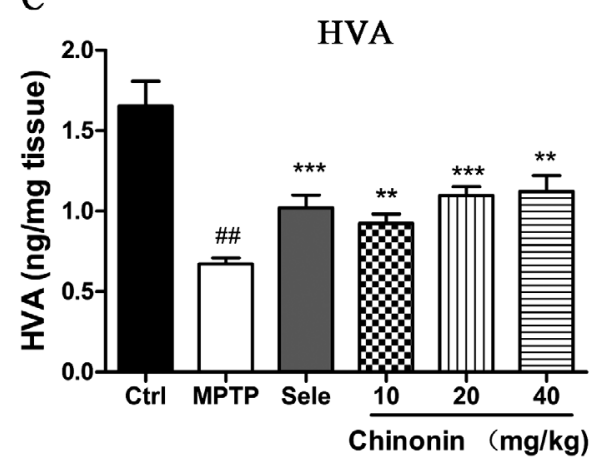

B

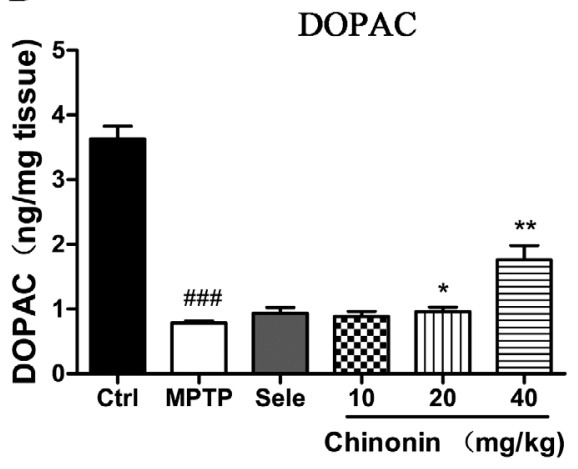

Fig. 5. Effect of Chinonin on MPTP-Induced Loss of Striatal Dopamine and Its Metabolites

Data were expressed as mean \pm S.D. $(n=6) .{ }^{\# \#} p<0.01$ and ${ }^{\# \# \#} p<0.001$ compared to control group; $* p<0.05$, ** $p<0.01$ and $* * * p<0.001$ compared to MPTP-treated group.

A

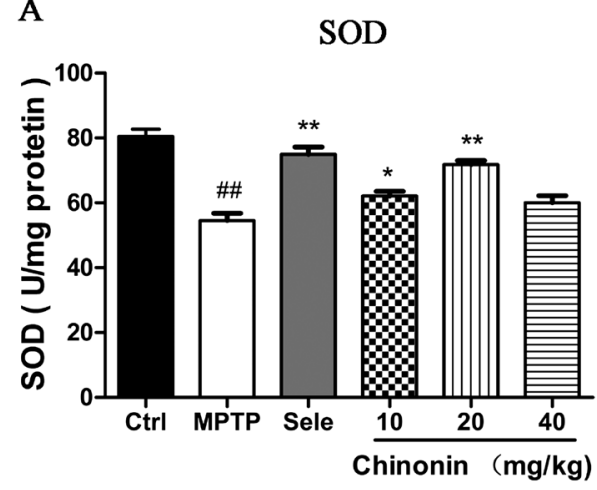

$\mathrm{C}$

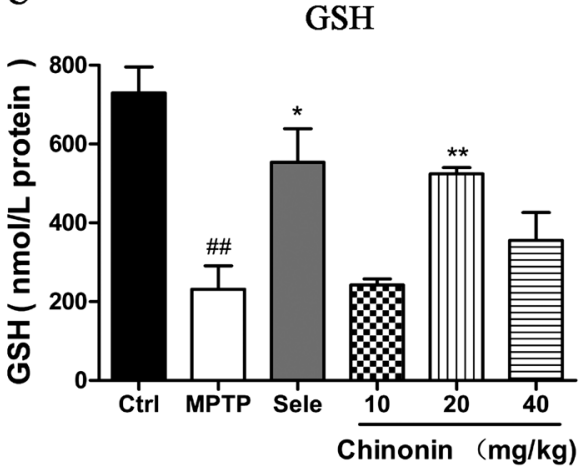

B

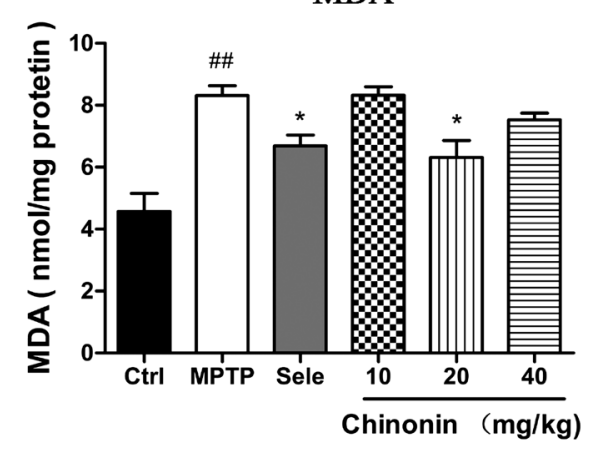

Fig. 6. Effects of Chinonin on the Levels of GSH, MDA, SOD in the Striatum of MPTP-Treated Mice

Data were expressed as mean \pm S.D. $(n=6) .{ }^{\#} p<0.01$ compared to control group; $* p<0.05$ and $* * p<0.01$ compared to MPTP-treated group.

highest activity. Chinonin is a natural polyphenols compound extracted from Anemarrhena asphodeloides which had antioxidative activity. As mentioned above, its anti-oxidative ac- tion might account much for its neuroprotection which caused the improvement of locomotor dysfunction. In some other researches, apocynin, another well studied natural antioxidant 
and its analogues were also observed to exert anti-oxidative effect in a dose dependent manner only within a certain range of concentration or dose, and showed a decrease of activity when exceeding the range. ${ }^{24,25)}$ Herein, $40 \mathrm{mg} / \mathrm{kg}$ might exceed the range so that to result in a reverse drop of action with the increase of dosage. The reason will be further studied.

Consistent with our findings, Kavitha found that chinonin attenuated the dopaminergic neurodegeneration in MPTPinduced C57BL/6 mice. ${ }^{9)}$ However, in Kavitha's experiment, mice were orally treated with chinonin for $14 \mathrm{~d}$ and from 10th day onwards MPTP (intraperitoneally (i.p.)) was injected for last $5 \mathrm{~d}$. The difference was that we adopted intraperitoneal injection which showed a better bioavailability than oral administration. Besides, to assess behavioral changes of mice, we adopted more advanced technology (catwalk and open-field) which was more repeatable, stable and scientific than traditional methods, such as swim test, hang test and so on. Generally, for some special diseases like PD, people take medicine for treatment after they are found ill rather than for prevention before diagnosis. In our study, the mice were treated with chinonin half an hour after MPTP injection which induced PDlike syndrome, and the neuroprotective effect of chinonin was confirmed. We will do more research including detailed mechanism research in animals model which has already developed PD by using the following protocol: the mice were injected MPTP $(30 \mathrm{mg} / \mathrm{kg})$ for 7 consecutive days and fed normally for $3 \mathrm{~d}$, then mice were injected chinonin for 14 consecutive days for efficacy observation.

In conclusion, chinonin reduced the death of dopaminergic neurons in substantial nigra, therefore reduced the depletion of dopamine and its metabolites and improved the behavioral disorder in MPTP-induced PD mice. The protective effects are possibly related to the anti-oxidative mechanisms. Thus, chinonin may be used as a potent neuroprotective agent against $\mathrm{PD}$ which is associated with oxidative stress.

Acknowledgment This study was supported by Guangdong province science and technology project (2010B011000006) to Sha Li.

\section{REFERENCES}

1) Dawson TM, Dawson VL. Molecular pathways of neurodegeneration in Parkinson's disease. Science, 302, 819-822 (2003).

2) Dauer W, Przedborski S. Parkinson's disease: mechanisms and models. Neuron, 39, 889-909 (2003).

3) Burns RS, Chiueh CC, Markey SP, Ebert MH, Jacobowitz DM, Kopin IJ. A primate model of parkinsonism: selective destruction of dopaminergic neurons in the pars compacta of the substantia nigra by $N$-methyl-4-phenyl-1,2,3,6-tetrahydropyridine. Proc. Natl. Acad. Sci. U.S.A., 80, 4546-4550 (1983).

4) Ito T, Suzuki K, Uchida K, Nakayama H. 1-Methyl-4-phenyl1,2,3,6-tetrahydropyridine (MPTP)-induced neuroblastic apoptosis in the subventricular zone is caused by 1-methy-4-phenylpiridinium $\left(\mathrm{MPP}^{+}\right)$converted from MPTP through MAO-B. Exp. Toxicol. Pathol., 64, 761-765 (2012).

5) Lee D-H, Kim C-S, Lee YJ. Astaxanthin protects against MPTP/ $\mathrm{MPP}^{+}$-induced mitochondrial dysfunction and ROS production0 in vivo and in vitro. Food Chem. Toxicol., 49, 271-280 (2011).

6) Jenner P. Oxidative stress in Parkinson's disease. Ann. Neurol., 53 (Suppl. 3), S26-S36, discussion, S36-S38 (2003).
7) Jenner P, Olanow CW. Oxidative stress and the pathogenesis of Parkinson's disease. Neurology, 47 (Suppl. 3), 161S-170S (1996).

8) Andreu GP, Delgado R, Velho JA, Curti C, Vercesi AE. Iron complexing activity of mangiferin, a naturally occurring glucosylxanthone, inhibits mitochondrial lipid peroxidation induced by $\mathrm{Fe}^{2+}$ citrate. Eur. J. Pharmacol., 513, 47-55 (2005).

9) Kavitha M, Nataraj J, Essa MM, Memon MA, Manivasagam T. Mangiferin attenuates MPTP induced dopaminergic neurodegeneration and improves motor impairment, redox balance and Bcl-2/Bax expression in experimental Parkinson's disease mice. Chem. Biol. Interact., 206, 239-247 (2013).

10) Abramov AY, Canevari L, Duchen MR. $\beta$-Amyloid peptides induce mitochondrial dysfunction and oxidative stress in astrocytes and death of neurons through activation of NADPH oxidase. J. Neurosci., 24, 565-575 (2004).

11) Martínez Sánchez G, Candelario-Jalil E, Giuliani A, León OS, Sam S, Delgado R, Núñez Sellés AJ. Mangifera indica L. extract (QF808) reduces ischaemia-induced neuronal loss and oxidative damage in the gerbil brain. Free Radic. Res., 35, 465-473 (2001).

12) Sánchez GM, Re L, Giuliani A, Núñez-Sellés AJ, Davison GP, León-Fernández O. Protective effects of Mangifera indica L. extract, mangiferin and selected antioxidants against TPA-induced biomolecules oxidation and peritoneal macrophage activation in mice. Pharmacol. Res., 42, 565-573 (2000).

13) Amazzal L, Lapôtre A, Quignon F, Bagrel D. Mangiferin protects against 1-methyl-4-phenylpyridinium toxicity mediated by oxidative stress in N2A cells. Neurosci. Lett., 418, 159-164 (2007).

14) Hamers FP, Lankhorst AJ, van Laar TJ, Veldhuis WB, Gispen WH. Automated quantitative gait analysis during overground locomotion in the rat: its application to spinal cord contusion and transection injuries. J. Neurotrauma, 18, 187-201 (2001).

15) Teng X, Sakai T, Liu L, Sakai R, Kaji R, Fukui K. Attenuation of MPTP-induced neurotoxicity and locomotor dysfunction in Nucling-deficient mice via suppression of the apoptosome pathway. J. Neurochem., 97, 1126-1135 (2006).

16) Vandeputte C, Taymans J-M, Casteels C, Coun F, Ni Y, Van Laere $\mathrm{K}$, Baekelandt V. Automated quantitative gait analysis in animal models of movement disorders. BMC Neurosci., 11, 92 (2010).

17) Jackson-Lewis V, Jakowec M, Burke RE, Przedborski S. Time course and morphology of dopaminergic neuronal death caused by the neurotoxin 1-methyl-4-phenyl-1,2,3,6-tetrahydropyridine. $\mathrm{Neu}$ rodegeneration, 4, 257-269 (1995).

18) Sankar SR, Manivasagam $T$, Krishnamurti A, Ramanathan $M$. The neuroprotective effect of Withania somnifera root extract in MPTP-intoxicated mice: An analysis of behavioral and biochemical varibles. Cell. Mol. Biol. Lett., 12, 473-481 (2007).

19) Guo B, Xu D, Duan H, Du J, Zhang Z, Lee S, Wang Y. Therapeutic effects of multifunctional tetramethylpyrazine nitrone on models of Parkinson's disease in vitro and in vivo. Biol. Pharm. Bull., 37, 274-285 (2014).

20) Barnham KJ, Masters CL, Bush AI. Neurodegenerative diseases and oxidative stress. Nat. Rev. Drug Discov., 3, 205-214 (2004).

21) Danielson SR, Andersen JK. Oxidative and nitrative protein modifications in Parkinson's disease. Free Radic. Biol. Med., 44, 1787-1794 (2008).

22) Tsang AH, Chung KK. Oxidative and nitrosative stress in Parkinson's disease. Biochim. Biophys. Acta, 1792, 643-650 (2009).

23) Jenner P. Oxidative mechanisms in nigral cell death in Parkinson's disease. Mov. Disord., 13 (Suppl. 1), 24-34 (1998).

24) Tang XN, Cairns B, Cairns N, Yenari MA. Apocynin improves outcome in experimental stroke with a narrow dose range. Neuroscience, 154, 556-562 (2008).

25) Lu X, Wan S, Jiang J, Jiang X, Yang W, Yu P, Xu L, Zhang Z, Zhang G, Shan L, Wang Y. Synthesis and biological evaluations of novel apocynin analogues. Eur. J. Med. Chem., 46, 2691-2698 (2011). 